\title{
Brugada Syndrome: Diagnosis and Management
}

\author{
Amit Vira, MD and Ehsanur Rahman, MD
}

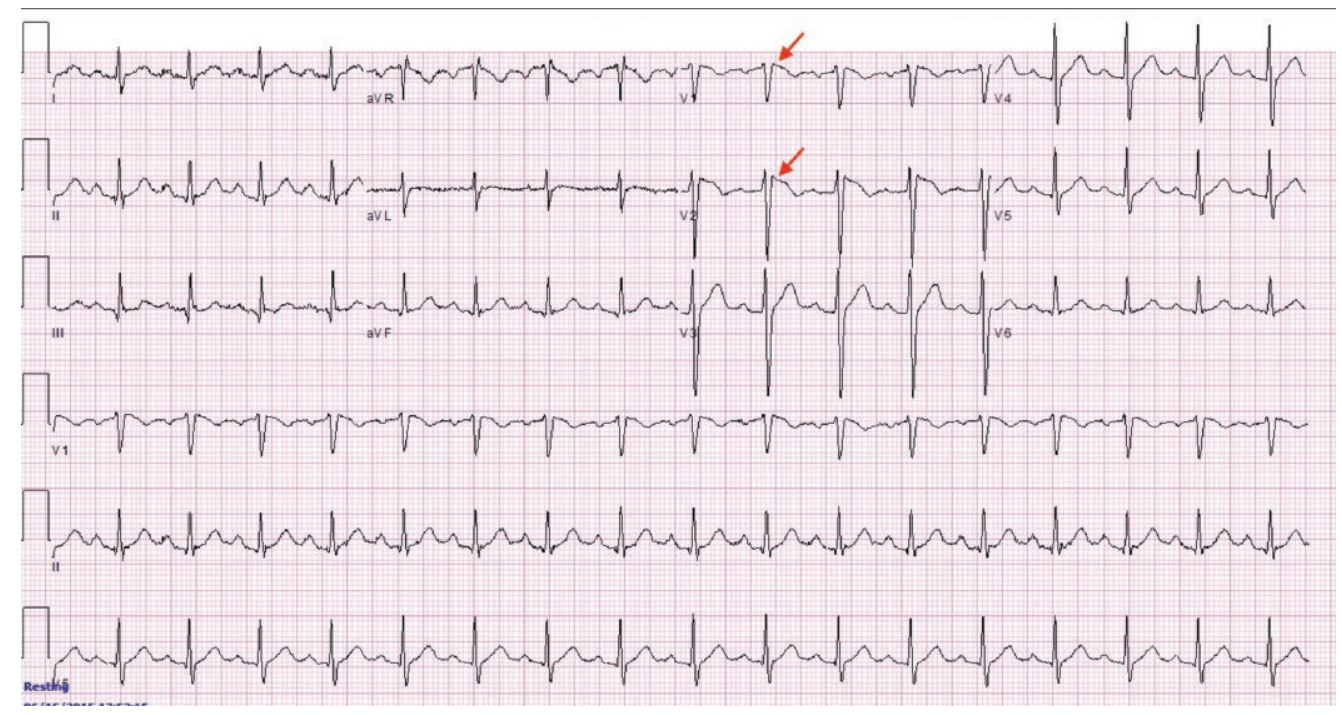

Figure 1. Type 1 Brugada pattern. 2-3 mm coved ST segment elevation in leads V1 and V2 followed by negative $T$ waves.

\section{INTRODUCTION}

The vast majority of cases of sudden cardiac arrest (SCA) are caused by ventricular tachyarrhythmias (VT), with most cases associated with structural heart disease. SCA in a structurally normal heart is an uncommon occurrence, most often occurring in the third decade of life and accounting for 5-15\% of total SCA cases ${ }^{1-2}$. Common causes include Brugada syndrome, congenital long QT syndrome, catecholaminergic polymorphic ventricular tachycardia, arrhythmogenic right ventricular cardiomyopathy, and idiopathic ventricular tachycardia ${ }^{1-3}$. The majority of these patients with a structurally normal heart are undiagnosed until presenting with sudden cardiac death. It is believed that cardiac arrest is the initial manifestation of nearly $50 \%$ of cases among those with structurally normal hearts 2

Here, we review a classic case of Brugada Syndrome, a common cause of sudden cardiac arrest in young adults, its diagnostic criteria, and management in a patient presenting for elective surgery.

\section{CASE PRESENTATION}

A 38-year-old male Iraq War combat veteran with a congenital cleft palate requiring multiple surgical repairs presents for cardiac evaluation when his preoperative electrocardiogram (ECG) showed new 2-3 mm coved ST elevation in leads V1 and V2 (Figure 1). On history, he reports multiple prior syncopal events while on active military service during extreme hot temperature conditions. All events were preceded by lightheadedness. He also reports a recent syncopal event 6 months prior to presentation that was preceded by palpitations causing a traumatic fall down staircases. He denies any prior cardiac workup. Review of systems was negative for chest pain, dyspnea, diaphoresis, dizziness, or flushing. Family history of cardiovascular disease was unknown as he is adopted. Social history was positive for a 20-pack-year history of cigarette use but negative for alcohol or illicit drugs. Physical exam was notable for a fistula involving the hard palate. His cardiopulmonary exam was normal. Laboratory studies included complete blood count, complete metabolic panel, thyroid stimulation hormone, urinalysis, and urine drug screen were all within normal limits. Transthoracic echocardiogram showed normal chamber size, thickness, and valves along with normal ventricular function.

\section{DIFFERENTIAL DIAGNOSIS}

When evaluating the cause of ST elevation on an ECG among young individuals without known cardiac disease or risk factors, the most common cause is benign early repolarization. However, it is important consider all other causes of ST elevation. These causes include ST elevation myocardial infarction (STEMI), pericarditis, bundle branch block, coronary vasospasm, ventricular aneurysm, left ventricular hypertrophy, and rarely, Brugada syndrome $e^{1.4}$.

Brugada syndrome is often referred to as a channelopathy and is linked to mutations in the cardiac sodium-channel gene causing a loss of the action potential in the right 
ventricular epicardium. While not completely understood, this creates a transmural voltage gradient that is thought to be responsible for the ST elevation in the right precordial leads and predisposes to ventricular arrhythmias ${ }^{5}$. Symptoms include history of VT, SCA, cardiac syncope, nocturnal agonal respiration, palpitations, or chest discomfort, which are not specific to the diagnosis of Brugada syndrome. These symptoms often occur at rest, during sleep, febrile states, cocaine use, or administration of class 1 antiarrhythmic drugs.

\section{DIAGNOSIS}

The diagnosis of Brugada syndrome requires characteristic ECG findings of a type 1 pattern. Type 1 Brugada pattern is defined by $\geq 2 \mathrm{~mm}$ coved ST elevation with negative $T$ wave in at least one of the right precordial leads (V1-V2) (Figure 2) spontaneously, or induced by provocative drug testing with intravenous class I antiarrhythmic drugs (i.e. flecainide, or procainamide) ${ }^{6}$. This differs from prior diagnostic criteria that also required evidence of documented sustained VT or cardiac syncope ${ }^{1}$. The change was based on recent evidence that individuals with the characteristic type 1 pattern, without the clinical symptoms, were still at high risk for future $\mathrm{SCA}^{6}$. Among patients with baseline type 2 ( $\geq$ $2 \mathrm{~mm}$ saddleback ST elevation) or type $3(\leq 2 \mathrm{~mm}$ saddleback ST elevation) on ECG, the diagnosis can be made if provocative testing with intravenous administration of class I antiarrhythmic drugs induce a type I ECG morphology (Figure 3) 6 . Provocative drug testing is only recommended in patients with previous symptoms suggestive of Brugada Syndrome. Asymptomatic nontype 1 ECG does not appear to have additional value for risk stratification. Additionally, genetic testing is not recommended in the absence of a type 1 ECG. However, genetic testing may be useful in patients with known family members with a positive genotype ${ }^{6}$.
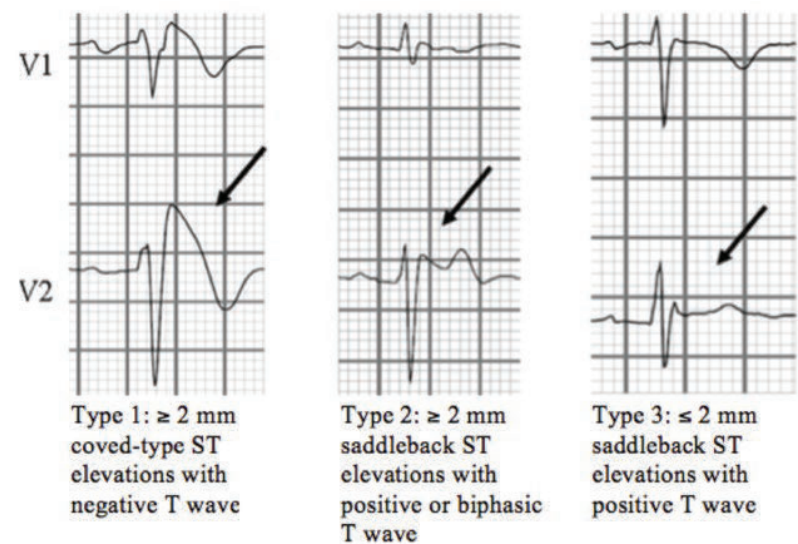

Figure 2. Characteristic Brugada electrocardiogram findings. $1{ }^{*}$ |mage modified from Wilde et al., 2002.

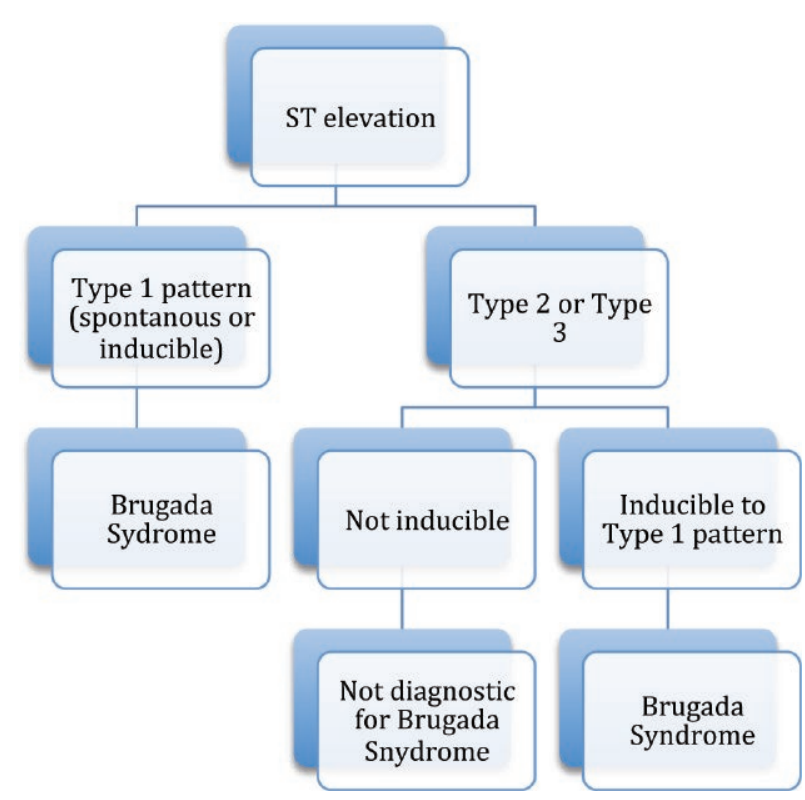

Figure 3. Type 1 is diagnostic for Brugada syndrome. Type 2 or Type 3 is nondiagnostic and additional drug challenge is required to unmask the Type 1 pattern.

\section{MANAGEMENT}

The diagnosis of Brugada syndrome is challenging, as often times SCA or cardiac syncope is the only presenting symptom. Given the high-risk for developing life-threatening ventricular arrhythmias and/or sudden cardiac death, an implantable cardioverter defibrillator (ICD) is the definite treatment ${ }^{1,5-7}$. The recommendation for an ICD is dependent on the patient's risk factors for future cardiac arrest, which includes a history of previous SCA, sustained VT, cardiac syncope, and a family history of Brugada syndrome or sudden cardiac death ${ }^{1,5-7}$. Patients with Brugada syndrome and a history of SCA or sustained $V T$ have the highest lifetime incidence for future SCA at $62 \%$. Thus, an ICD placement is recommended in these patients (class I recommendation) (Figure 4$)^{6}$. For patients with Brugada syndrome and a history of cardiac syncope, an ICD placement is also recommended (class 1 la recommendation), given that $19 \%$ of patients develops a future SCA (Figure 4$)^{6}$. Risk-stratification of patients without a history of SCA, sustained VT, or cardiac syncope has remained controversial. It has been proposed that additional risk stratification with an electrophysiologic study may help identify high-risk patients. However, several recent studies, including the PRELUDE registry, have shown that inducible VT is unable to identify high-risk patients ${ }^{6}$. Thus, it is a class IIb recommendation for ICD placement for primary prevention in patients with type 1 pattern and 


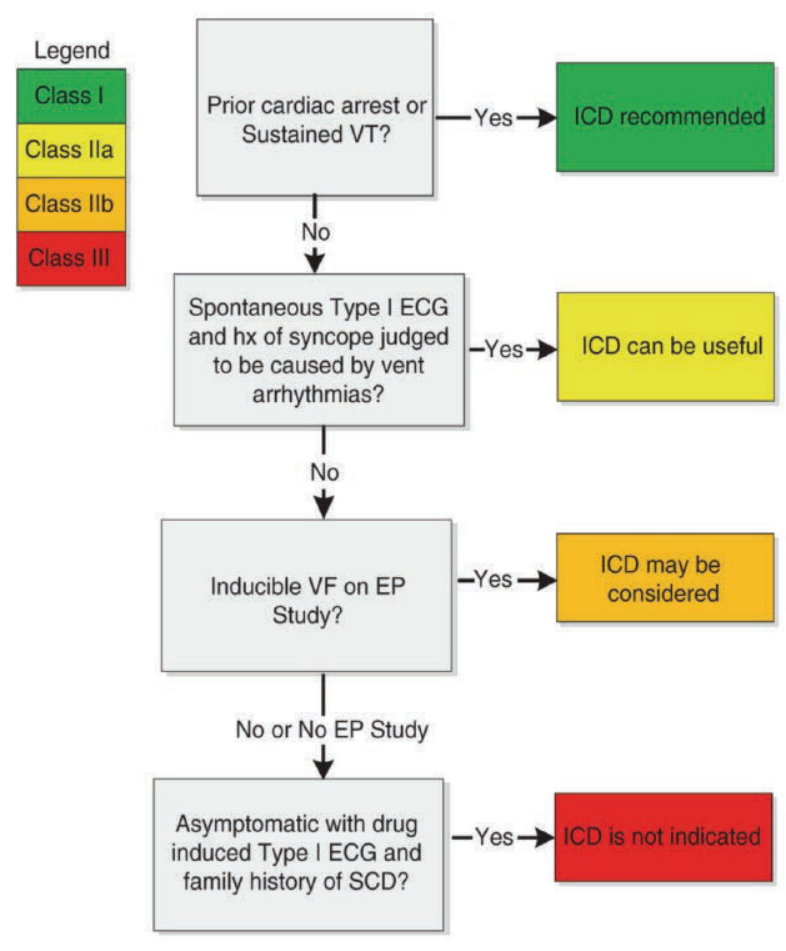

Figure 4. Recommendations for ICD placement for Brugada syndrome. ${ }^{6}$ *Image modified from Priori et al., 2013.

inducible VT on electrophysiologic study (Figure 4)6. In asymptomatic patients with a drug-induced type 1 pattern along with a family history of SCA, an ICD placement is not recommended for primary prevention (class III recommendation) (Figure 4) ${ }^{6}$. Additionally, patients should undergo lifestyle changes, which include avoidance of provocative drugs (www. BrugadaDrugs. org), avoidance of excessive alcohol, and treatment of fevers with antipyretic drugs (class I recommendation) ${ }^{6}$.

\section{OUTCOME}

The patient in the aforementioned case was diagnosed with Brugada syndrome given the presence of type 1 Brugada pattern on his ECG. Due to his extensive history of syncope, there was high suspicion for ventricular tachyarrhythmias as the cause of his syncope. Thus, patient was deemed at high risk for future SCA and was referred for ICD placement.

\section{KEY POINTS}

- Suspect Brugada syndrome in young patients with a history of SCA, sustained VT, cardiac syncope, or family history of Brugada in the absence of structurally heart disease.

- Brugada syndrome is diagnosed by characteristic ST segment elevation in the right precordial leads (Type 1 Brugada pattern).

- High-risk patients (previous SCA, sustained VT, or cardiac syncope) should be recommended for ICD placement.

- The definitive treatment is placement of an ICD

\section{REFERENCES}

1. Arthur A.M. Wilde, Charles Antzelevitch, Martin Borggrefe, Josep Brugada Ramón Brugada, Pedro Brugada, Domenico Corrado, Richard N.W. Hauer, Robert S. Kass, Koonlawee Nademanee, Silvia G. Priori and Jeffrey A. Towbin Proposed diagnostic criteria for the Brugada syndrome: consensus report. Circulation. 2002; 106 (19): 2514-19

2. Chugh, S. Kelly, K. Titus, J. Sudden Cardiac Death With Apparently Normal Heart. Circulation. 2000;102:649-654

3. Modi S. Krahn A. Sudden Cardiac Arrest Without Overt Heart Disease. Circulation. 2011:123:2994-08

4. Wang K, Asinger R, Marriott H. ST-Segment Elevation in Conditions Other Than Acute Myocardial Infarction. The New England Journal of Medicine. 2003; 349:2128-35

5. Kurita T, Shimizu W, Inagaki M, Suyama K, Taguchi A, Satomi K, Aihara N, Kamakura S, Kobayashi J, Kosakai Y. The electrophysiologic mechanism of ST-segment elevation in Brugada syndrome. J Am Coll Cardiol. 2002 Jul 17;40 (2):330-4.

6. Priori SG, Wilde AA, Horie M, Cho Y, Behr ER, Berul C, Blom N, Brugada J, Chiang CE, Huikuri H, Kannankeril P, Krahn A, Leenhardt A, Moss A, Schwartz PJ, Shimizu W, Tomaselli G, Tracy C. 2013 HRS/EHRA/APHRS Expert Consensus Statement on the Diagnosis and Management of Patients with Inherited Primary Arrhythmia Syndromes. Heart Rhythm. 2013 Dec; 10 (12): 1932-1963.

7. Priori SG, Gasparini M, Napolitano C, Della Bella P, Ottonelli AG, Sassone B, Giordano U, Pappone C, Mascioli G, Rossetti G, De Nardis R, Colombo M. Risk stratification in Brugada syndrome: results of the PRELUDE (PRogrammed ELectrical stimUlation preDictive valuE) registry. J Am Coll Cardiol. 2012 Jan 3; 59 (1): $37-45$

8. Brugada J, Brugada R, Antzelevitch C, Towbin J, Nademanee K, Brugada P. Long-term follow-up of individuals with the electrocardiographic pattern of right bundle-branch block and ST-segment elevation in precordial leads V1 to V3. Circulation. 2002 Jan 1; 105 (1): 73-78 